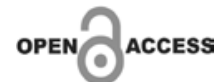

Correspondence addresses:

Dr. Carlos Ramon Mendes

proctoramon@hotmail.com

Received: August 9, 2021

Revised: August 18, 2021

Accepted: August 21, 2021

Published: September 30, 2021

Data Availability Statement: All relevant data are within the paper and its Supporting Information files.

Funding: This work was the result of authors' initiative. There was no support of research or publication funds.

Competing interests: The authors have declared that no competing interests exist.

\section{RESUMO DE ARTIGO}

\title{
Estudo Multicêntrico Brasileiro e Argentino no Tratamento Cirúrgico do Cisto Pilonidal por Técnica Minimamente Invasiva
}

\author{
Brazilian and Argentinean Multicentric Study in the Surgical \\ Minimally Invasive Treatment of Pilonidal Cyst
}

\author{
Carlos Ramon Silveira Mendes ${ }^{1 *}$, Luciano Santana de Miranda Ferreira ${ }^{1}$, \\ Leonardo Salim ${ }^{2}$ \\ ${ }^{1}$ Departamento de Coloproctologia, Hospital Santa Izabel, Salvador, Bahia, Brazil; \\ ${ }^{2}$ Parque Clinic, Coloproctologia Rosario, Rosario, Argentina
}

Racional: $O$ cisto pilonidal é infecção da pele e do tecido subcutâneo, secundário à inflamação crônica, com maior frequência na região sacrococcígea, e associado à presença de pelos nesta região. $O$ tratamento é eminentemente cirúrgico. Objetivo: Demonstrar os resultados do tratamento endoscópico de cisto pilonidal. Método: Estudo prospectivo, com 67 pacientes que tiveram como indicação cirúrgica o diagnóstico de cisto pilonidal. Os equipamentos utilizados foram o fistuloscópio Meinero, um obturador, um eletrodo monopolar, uma escova e pinça endoscópica. Resultados: Dos 67 pacientes $67 \%$ $(n=45)$ eram homens e $33 \%(n=22)$ mulheres, com média de idade de 25 anos (17-45). O tempo cirúrgico teve com média 40 min (20-120) e o tempo médio de cicatrização de quatro semanas (3-12). Complicações cirúrgicas ocorreram em $7 \%$ da amostra $(n=5)$ e recidivas da doença em $9 \%(n=6)$. Conclusão: $O$ tratamento endoscópico do cisto pilonidal é viável e apresenta bons resultados cirúrgicos.

Background: The pilonidal cyst is an infection of the skin and the subcutaneous tissue, secondary to a chronic inflammation with a greater frequency in the sacrococcygeal region, and associated to the presence of hair. The treatment is eminently surgical. Aim: To demonstrate the endoscopic treatment of pilonidal cyst. Method: Prospective study with 67 patients who had as surgical indication the diagnosis of pilonidal cyst. They were submitted to a surgical procedure from June 2014 to March 2018. The equipment used was the Meinero fistuloscope, a shutter, a monopolar electrode, a brush and endoscopic forceps. Results: Of the 67 patients, $67 \%(n=45)$ were male and $33 \%(n=22)$ female, with a mean age of 25 years (17-45). Surgical time in average was 40 min (20-120) and mean healing time of four weeks (3-12). Surgical complications were presented in $7 \%$ cases $(n=5)$ and recurrences in $9 \%(n=6)$. Conclusion: The endoscopic treatment of the pilonidal cyst is feasible and presents good surgical results.

\section{Copyright}

(C) 2021 by Santa Casa de Misericórdia da Bahia. All rights reserved. ISSN: $2526-5563$

DOI: 10.35753

Resumo de Artigo: Mendes CRS, Ferreira LSM, Salim L. Estudo multicêntrico brasileiro e argentino no tratamento cirúrgico do cisto pilonidal por técnica minimamente invasiva. ABCD Arq Bras Cir Dig. 2019;32(3):e1447. DOI: /10.1590/0102-672020190001e1447. 


\section{Introdução}

O cisto pilonidal é doença da pele e tecido subcutâneo, ocorrendo predominantemente em homens com idade média de 30 anos e incidência de 26 casos por $100.000 .{ }^{1}$ A doença é caracterizada por quadros assintomáticos até lesões dolorosas localizadas na região sacrococcígea. ${ }^{2}$ A causa não está completamente elucidada; no entanto, alguns fatores de risco como a obesidade, higiene pessoal inadequada, histórico familiar e longos períodos em posição sentada estão associados a maior ocorrência da doença. ${ }^{3}$

O tratamento é cirúrgico, verificando-se grande diversidade de técnicas. As técnicas menos invasivas constituem-se como alternativa aos métodos de excisão cirúrgica, apresentando vantagens como menor dor no período pósoperatório, retorno precoce do paciente às suas atividades e menores cicatrizes. ${ }^{4}$

O tratamento endoscópico minimamente invasivo do cisto pilonidal (EPSiT) proposto por Meinero e colaboradores ${ }^{5}$ baseia-se no tratamento da fístula anal por vídeo. A técnica é subdividida em duas etapas caracterizadas pela fase do diagnóstico e fase operatória. A diagnóstica destina-se a identificar e caracterizar o cisto bem como identificar cavidades secundários contendo abcessos. ${ }^{1,5}$

O objetivo deste trabalho foi demonstrar a efetividade do tratamento endoscópico minimamente invasivo no tratamento cirúrgico do cisto pilonidal.

\section{Métodos}

\section{Características do Estudo}

Trata-se de um estudo prospectivo, realizado no período de junho de 2014 a março de 2018, compreendendo pacientes com quadros sintomáticos de cisto pilonidal que deram entrada no Hospital Provincial de Rosário em Santa Fé na Argentina e no Hospital Santa Izabel em Salvador no Brasil.

\section{Técnica Cirúrgica}

A técnica endoscópica foi executada conforme o método idealizado por Meinero e colaboradores (2014), ${ }^{5}$ utilizando o fistuloscópio de Meinero (Karl Storz GmbH - Tuttlingen, Alemanha, Figura 1A). Os pacientes foram submetidos à raquianestesia e o procedimento teve início em posição pronada.

$\mathrm{O}$ procedimento tem início com a fase de identificação e caracterização da extensão do cisto, bem como de cavidades secundárias por meio da inserção do fistuloscópio com infusão de glicina ou manitol na abertura espontânea do cisto (Figura 1B). A seguir, o cisto é aberto com auxílio de jato destes líquidos a fim de identificar pelos e eliminar tecidos danificados. Os pelos presentes no trato são removidos com auxílio do fórceps e o tecido granular é tratado por meio do eletrodo monopolar. ${ }^{4}$ Todo tecido granular é destruído e removido, os pelos retirados e, em seguida, o trato é limpo e ficando cavidade aberta para facilitar a drenagem (Figuras 1C e 1D).

\section{Resultados}

As características dos pacientes e os resultados obtidos estão descritos na Tabela 1.

Entre o período do estudo foram selecionados 67 pacientes, dos quais $67 \%$ eram homens, com idade média de 31 anos (17-45). O procedimento

Tabela 1. Características dos pacientes e resultados obtidos.

\begin{tabular}{lc}
\hline Variável & N (\%) \\
\hline Idade (anos) & $31 \pm 14$ \\
Gênero & \\
$\quad$ Masculino & $45(67)$ \\
$\quad$ Feminino & $22(33)$ \\
Tempo médio da operação & $40 \mathrm{~min}$ \\
Tempo médio de cicatrização & $4 \pm 4$ \\
(semanas) & \\
Complicações & $5(7)$ \\
Recidivas & $6(9)$ \\
\hline
\end{tabular}


Figura 1. A) Fistuloscópio Karl-Storz. B) Exploração do cisto. C) Aspecto cirúrgico final do procedimento. D) Pós-operatório após 15 dias.

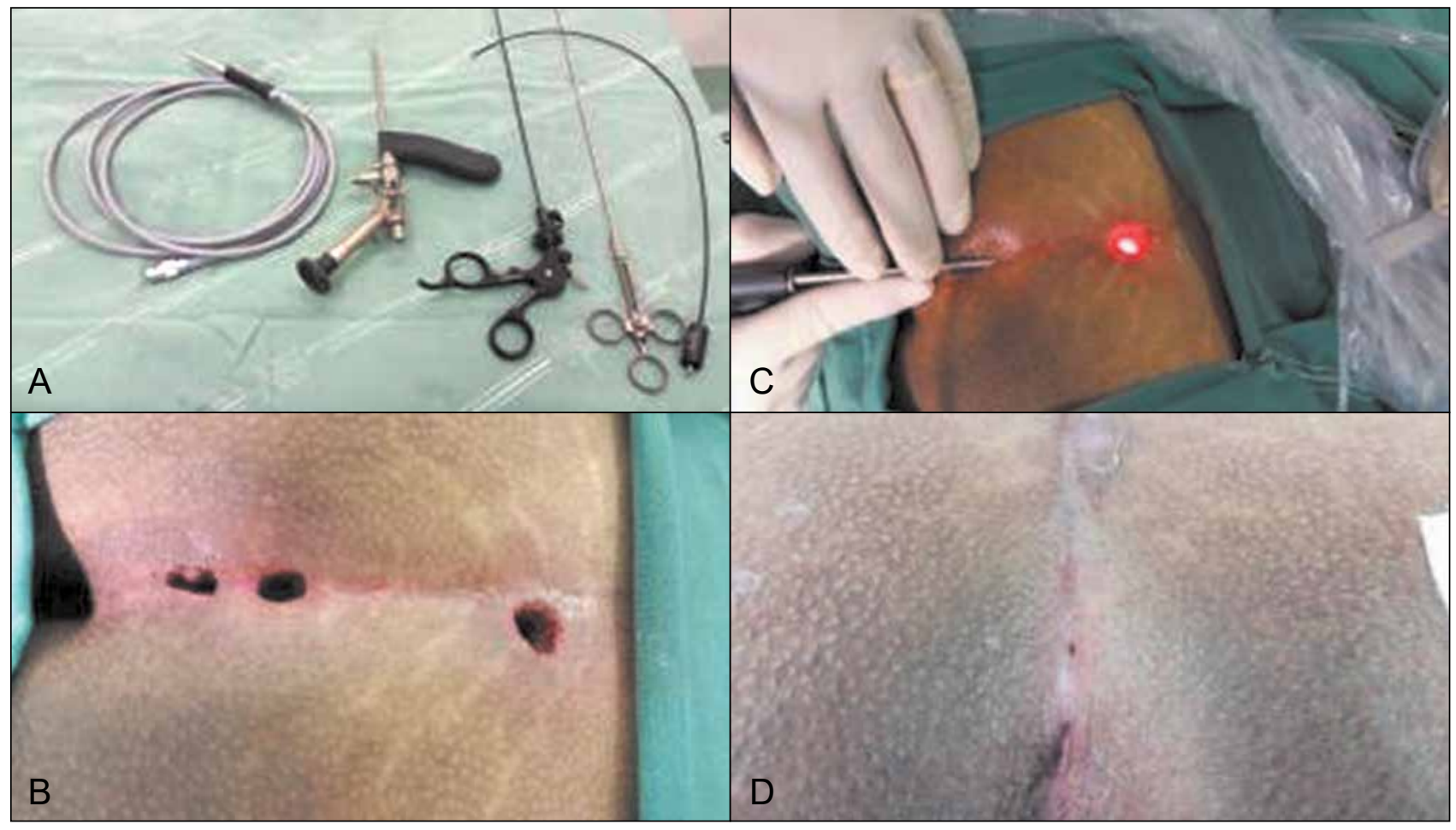

cirúrgico durou em média $40 \mathrm{~min}$; os pacientes receberam alta no mesmo dia após a realização do procedimento e o tempo médio de cicatrização foi de quatro semanas (3-12).

Complicações maiores não foram observadas; no entanto, cinco casos apresentaram complicações menores tais como sangramento e dor por período prolongado. A taxa de cura foi de $91 \%$, verificando-se que cinco pacientes apresentaram recidiva e nenhum outro caso de falha no tratamento ou persistência do cisto.

\section{Discussão}

Embora o cisto pilonidal tenha sido descrito há mais de 150 anos, ${ }^{2}$ e apesar do tratamento ser majoritariamente cirúrgico, existem diversas técnicas cirúrgicas descritas que incluem a excisão do cisto, técnicas de retalho e, mais recentemente, técnicas minimamente invasivas. ${ }^{6}$ Stauffer e colaboradores $(2018)^{7}$ por meio de revisão sistemática identificaram pelo menos 14 estratégias terapêuticas para o tratamento do cisto e sua recorrência.

Meinero e colaboradores ${ }^{5}$ empregaram a técnica de tratamento da fístula anal assistida por vídeo para o tratamento da doença pilonidal, visando evitar um dos maiores inconvenientes dos procedimentos que objetivam a remoção da área infectada por excisão, que é a cicatrização da ferida cirúrgica, aberta ou fechada. Em ambos os casos, o período pós-operatório necessita da realização de curativos, o que aumenta o tempo necessário para a cura, além de causar dor. ${ }^{5,8}$

A técnica endoscópica para o tratamento do cisto pilonidal ocasiona menor dor pósoperatória, rápida cura e menor tempo para o retorno às atividades diárias. Além disso, ela apresenta grande eficiência nos casos de recorrência do cisto.

Essa técnica apresenta vantagens em relação às outras mais invasivas que são realizadas 
às cegas, o que explica a maior ocorrência de recidivas nesses casos. O tratamento endoscópico é favorecido pela observação do interior do cisto, possibilitando ao cirurgião identificar o local dos pelos, bem como de tecidos danificados, contribuindo para a maior taxa de sucesso. ${ }^{6}$

O tempo necessário para cicatrização após a técnica EPSiT foi menor do que outras abordagens cirúrgicas durante o estudo. Segundo Bernier e colaboradores (2015), ${ }^{9}$ cerca de 10\%-30\% dos pacientes submetidos a mais de um tratamento cirúrgico poderão evoluir para cronicidade. $\mathrm{O}$ tratamento endoscópico está associado a uma baixa ocorrência de recorrência, ${ }^{10}$ sobretudo se comparado às técnicas menos invasivas. ${ }^{7}$

Dentre os diversos tratamentos para o cisto pilonidal, a técnica EPSiT apresenta-se como alternativa segura; no entanto, sua utilização depende de equipamento específico, o que pode limitá-la. ${ }^{4}$

Esta técnica empregada com pioneirismo no Brasil e na Argentina, representa alternativa segura e reprodutível para o tratamento do cisto pilonidal, permitindo ainda que o paciente retome suas atividades diárias em curto período de tempo. Além disto, esteticamente apresenta-se superior às demais, pois resulta em poucas cicatrizes.

\section{Conclusão}

O tratamento endoscópico para o tratamento do cisto pilonidal demonstrou grande segurança e eficiência. A técnica oferece benefícios como bons resultados, tempo reduzido de recuperação e baixo índice de complicações.

\section{Referências}

1. Tien T, Athem R, Arulampalam T. Outcomes of endoscopic pilonidal sinus treatment (EPSiT): a systematic review. Tech Coloproctol 2018; 22: 325331.

2. Chintapatla S, Safrani N, Kumar S, Haboubi N. Sacrococcygeal pilonidal sinus: historical review, pathological insight and surgical options. Tech Coloproctol2003; 7: 3-8.

3. Tas, Sukru et al. Management of flap dehiscense after Limberg procedure for recurrent pilonidal disease by negative pressure wound therapy (NPWT). ABCD, Arq. Bras. Cir. Dig., Mar 2017;30(1): 73-74.

4. Mendes CRS, Ferreira LSM, Sapucaia RA, Lima MA. Endoscopic pilonidal sinus treatment (E.P.Si.T): a minimally invasive approach. J. Coloproctol 2015; 35: $72-75$.

5. Meinero P, Mori L, Gasloli G. Endoscopic pilonidal sinus treatment (EPSiT). Tech Coloproctol. 2014; 18: 389-392.

6. Emile SH, Elfeki H, Shalaby M, Sakr A, Giaccaglia V, Sileri P, et al. Endoscopic pilonidal sinus treatment: a systematic review and meta-analysis. Surg Endosc. 2018; 1-9.

7. Stauffer VK, Luedi MM, Kauf $P$, Schmid M, Dieckmann M, Weiferich K, et al. Common surgical procedures in pilonidal sinus disease: A metaanalysis, merged data analysis, and comprehensive study on recurrence. Sci Rep. 2018; 15: 3058.

8. Giarratano G, Toscana C, Shalaby M, Buonomo O, Petrella G, Sileri P. Endoscopic pilonidal sinus treatment: Long-term results of a prospective series. J. Soc. Laparendosc. Surgeons. 2017; 21: e2017.0043

9. Bernier GV, Johnson EK, Maykel JA, Steele SR. Reoperative surgery for pilonidal disease. Sem. Colon Rectal Surgery. 2015; 26: 211-217.

10. Milone M, Musella M, Sardo A, Bifulco G, Salvatore G, Fernandez L, et al. Video-assisted ablation of pilonidal sinus: a new minimally invasive treatment - a pilot study. Surgery2014; 155: 562-566. 